\title{
Efecto de la suplementación combinada con hierro y zinc sobre el estado nutricional de cobre en mujeres de edad reproductiva
}

\author{
Effect of a combined iron \\ and zinc supplementation on \\ copper status in women \\ of childbearing age
}

\begin{abstract}
Iron ( $\mathrm{Fe})$, copper (Cu) and zinc ( $\mathrm{Zn}$ ) deficiency coexist in developing countries. Supplementation and fortification programs with combined micronutrients have been implemented, but possible negative interactions of these minerals absorption should be considered. The aim of this study was to evaluate the effect of a daily supplementation with $30 \mathrm{mg}$ of Fe alone or in combination with $30 \mathrm{mg}$ of $\mathrm{Zn}$ on Cu status in Chilean women of childbearing age. This was a randomized, double-blind, placebo-controlled trial, in which 81 women aged $18-45 y$ were randomly assigned to receive either a daily single dose of $30 \mathrm{mg}$ of Fe or this mineral combined with 30mg of $\mathrm{Zn}$ or placebo. The concentration of serum copper (CuS), ceruloplasmin (Cp mass) ceruloplasmin activity (Cp activity) and specific activity (Cp activity/Cp mass) were evaluated at baseline and at the end of the study. The daily combined Fe and Zn supplementation significantly decreased CuS concentration (2-way ANOVA for repeated measures $p<0,01$ ), but Fe alone did not affect $\mathrm{Cu}$ status. Fe with Zn supplementation did not affect $C p$ mass, Cp activity and specific activity. In conclusion, combined supplementation of Fe and $\mathrm{Zn}$ in doses of 30mg each, significantly decreases CuS concentration.

Key words: Copper, iron, zinc, supplementation, women of childbearing.
\end{abstract}

Angélica Borja-Magno María F Mujica - Coopman Fernando Pizarro Aguirre Manuel Olivares Grohnert

Laboratorio de Micronutrientes Instituto de Nutrición y Tecnología de Alimentos (INTA), Universidad de Chile. Santiagp, Chile.

Este trabajo fue recibido el 15 de Abril de 2014 y aceptado para ser publicado el 11 de Noviembre de 2014.

conoce con exactitud su magnitud (4). Estas deficiencias no se presentan de forma aislada, sino que coexisten $(5,6)$ por tal motivo se han implementado estrategias de suplementación y fortificación de alimentos con estos micronutrientes para su prevención y tratamiento $(3,7)$. Sin embargo, existe la preocupación de una posible interacción negativa entre $\mathrm{Cu}$, Fe y Zn a nivel absortivo, que pueda alterar el estado nutricional de esos micronutrientes (8).

Estudios en humanos han mostrado resultados contradictorios acerca de la interacción entre estos minerales. Con respecto a la interacción de Fe y Cu se ha observado en lactantes que la suplementación con Fe puede disminuir la actividad Cu-Zn superóxido dismutasa (SOD1), no así el cobre sérico (CuS) (9).A su vez, en adultos se demostró que la suplementación con Fe no afectaba la absorción de Cu (10).Por lo 
tanto aún no existe evidencia concluyente del efecto negativo del Fe sobre el estado nutricional de $\mathrm{Cu}$ (11).

En cuanto a la interacción de Zn y Cu, el International Zinc Nutrition Consultative Group (IZiNCG) reportó que en adultos, dosis de suplementación entre 25 y $35 \mathrm{mg}$ de $\mathrm{Zn}$, no alteran el estado nutricional de $\mathrm{Cu}$, pero que dosis entre 100 a 300mg de $\mathrm{Zn}$ pueden inducir anemia por deficiencia de $\mathrm{Cu}$ (3). A su vez, un estudio en adolescentes y otro en adultos mayores mostraron que dosis más bajas de suplementación (22mg o 20mg de Zn) disminuían las concentraciones de CuS $(12,13)$. Por lo tanto, se necesita más evidencia para dilucidar esta discrepancia.

El objetivo de este estudio fue evaluar el efecto de la suplementación diaria durante 3 meses con $30 \mathrm{mg}$ de Fe como sulfato ferroso solo o combinado con $30 \mathrm{mg} \mathrm{Zn}$ como de sulfato de $\mathrm{Zn}$ sobre el estado nutricional de Cu en mujeres chilenas de edad reproductiva.

\section{MATERIAL Y MÉTODOS}

$$
\text { Sujetos }
$$

Se seleccionaron 87 mujeres de edad reproductiva, aparentemente sanas según su historia clínica, que no hubieran consumido suplementos de minerales seis meses previos al estudio, que no estuvieran embarazadas ni amamantando. Todas procedentes de la comuna de Macul, Santiago, Chile (Comuna de nivel socioeconómico medio-bajo).

\section{Diseño del estudio}

Este estudio fue un ensayo clínico, doble ciego, aleatorizado y controlado con placebo. Mediante una generación aleatoria de números en el programa Excel (Windows Microsoft 2010) se asignó a las participantes para recibir diariamente, por un período de 3 meses, un suplemento con 30mg de $\mathrm{Fe}$ elemental, como sulfato ferroso, o el mismo combinado con $30 \mathrm{mg}$ de $\mathrm{Zn}$ elemental como sulfato de zinc o un placebo. Los suplementos fueron suministrados en estómago vacío (1 hora antes o 3 horas después de las comidas). El personal del estudio en terreno diariamente lo suministró a las participantes y verificó su consumo. Las cápsulas fueron idénticas en apariencia, y fueron preparados en una farmacia local (Farmacias Cruz Verde, Santiago, Chile).

\section{Consideraciones éticas}

Las mujeres participantes del estudio, firmaron un consentimiento informado. Esta investigación fue aprobada por el Comité de Ética para la Investigación en humanos del Instituto de Nutrición y Tecnología de Alimentos (INTA).

Evaluación antropométrica y de ingesta dietética

Se evaluó el peso y talla de las participantes utilizando una balanza ( $0.1 \mathrm{~kg}$ de precisión) y tallímetro ( $1 \mathrm{~cm}$ de precisión) marca SECA, por técnicas descritas (14). Las participantes fueron clasificadas de acuerdo al criterio de la Organización Mundial de la Salud de acuerdo a su índice de masa corporal (IMC), con bajo peso (IMC >18.5 kg/m²), estado nutricional normal (IMC 18,5-24,9 kg/m²), sobrepeso (IMC 25,0 - 29,9 $\left.\mathrm{kg} / \mathrm{m}^{2}\right)$ y obesidad $\left(I M C \geq 30 \mathrm{~kg} / \mathrm{m}^{2}\right)$.

Se evaluó la ingesta dietética de las participantes al inicio $y$ al final del estudio utilizando una encuesta de frecuencia de consumo de alimentos (EFC). La ingesta alimentaria fue convertida en datos de ingesta de nutrientes utilizando la Tabla de Composición de Alimentos de Chile (15), y en caso de ser necesario se complementó con datos de los alimentos comúnmente consumidos en Chile (16) y con la base de datos nacionales de nutrientes estándar de referencia del Departamento de Agricultura de Estados Unidos (USDA) (17). La adecuación de la ingesta de micronutrientes se evaluó según el Requerimiento Promedio Estimado (EAR) (18).

\section{Parámetros hematológicos}

Al inicio y al final del estudio, se obtuvo en ayunas $15 \mathrm{ml}$ de sangre venosa, entre las 8 y 9 am. Se determinaron las concentraciones de cobre sérico (CuS) (Espectrómetro de absorción atómica, PerkinElmer Life and Analytic Sciencice modelo 2280, Norwalk, CT, USA), ceruloplasmina proteína inmunoreactiva (Cp masa) (Array Protein System, Beckman Instruments Inc., Brea, CA, USA), actividad de la ceruloplasmina (Cp actividad) (Método $p$-phenylenediamine oxidasa) (19) y la actividad específica de la ceruloplasmina (índice Cp actividad/ Cp masa). La deficiencia de Cu se definió como CuS $<80 \mu \mathrm{g} / \mathrm{dl}$, Cp masa <200mg/L (20) o Cp actividad <150mg/L (19). La deficiencia de Cu se evaluó con éstos indicadores al inicio y después de la intervención.

\section{Análisis estadístico}

El tamaño muestral se calculó considerando un cambio de $20 \mu \mathrm{g} / \mathrm{dl}$ de CuS en el mismo sujeto después de la suplementación, una desviación estándar de $23 \mu \mathrm{g} / \mathrm{dl}$ (21) con un nivel de significación de 0,05 y un poder del 80\%. Se consideraron 28 sujetos por grupo, haciendo un total de 84 participantes, pero finalmente, se incluyeron 87 mujeres.

Las variables con distribución normal fueron expresadas en promedios y desviación estándar (DE). Dado que las variables Cp masa y actividad no presentaron distribución normal se transformaron a su logaritmo natural y posteriormente se calculó el anti-logaritmo y se expresaron en promedio geométrico y rango $\pm 1 \mathrm{DE}$. El CuS fue transformado a su valor recíproco para el análisis y fue expresado en promedio y DE. Se realizó el test estadístico ANOVA de dos vías para medidas repetidas, para comparar el efecto del tiempo (antes vs después) y tipo de tratamiento sobre el estado nutricional de Cu y, ANOVA de una vía, para comparar las diferencias entre los valores iniciales y finales de acuerdo al tipo de tratamiento. Se utilizó el test post-hoc de Sheffé cuando hubo diferencias significativas. Se utilizó el programa estadístico STATA 12,0, StataCorp, College Station, TX, USA. Todas las comparaciones tuvieron un nivel de significación de 0,05.

\section{RESULTADOS}

Se evaluaron 87 mujeres de edad reproductiva con edad promedio de 33,2 años. Un total de 6 participantes abandonaron el estudio, de ellas, 3 lo hicieron por molestias digestivas, 1 fue excluida por comenzar un tratamiento con sulfato ferroso por anemia y 2 no se encontraban disponibles para recibir el suplemento. De las 81 mujeres que completaron el estudio, veintisiete recibieron diariamente durante tres meses un placebo, veintiocho recibieron el suplemento de Fe, y veintiséis el suplemento con la combinación de Fe y Zn (figura 1). Las características generales de las participantes se muestran en la tabla 1. No se encontraron diferencias significativas en la edad y parámetros antropométricos al comparar por tipo de tratamiento y tiempo (antes vs después)

Los indicadores del estatus de $\mathrm{Cu}$ se presentan en la tabla 2. CuS, Cp masa y actividad específica de Cp mostraron un efecto significativo respecto al tiempo (antes vs después) (ANOVA de 2 vías para medidas repetidas, $p<0,05$ ), no así la $C p$ actividad. Se observó una interacción significativa entre el tiempo y el tipo de tratamiento sobre CuS (ANOVA 
Medidas repetidas $\mathrm{p}<0,01)$. El test post-hoc mostró que el grupo suplementado con Fe y Zn tuvo concentraciones de CuS significativamente menores al comparar con el grupo que recibió Fe y placebo (figura 2).

Al inicio del estudio, la prevalencia de deficiencia de Cu según CuS fue de 1,2\% $(n=1)$ en el grupo suplementado con Fe y $\mathrm{Zn}$. No se encontró deficiencia de $\mathrm{Cu}$ medida por Cp masa. El 2,5\% ( $n=2), 1,2 \%(n=1), 4,9 \%(n=4)$ del grupo control, suplementado con Fe y Fe con Zn presentaron deficiencia según la $\mathrm{Cp}$ actividad, respectivamente. Al finalizar el estudio, 2,5\% $(n=2)$ de las participantes presentaron deficiencia de CuS en el grupo suplementado con Fe y Zn. No se encontró deficiencia según la $C p$ masa. El 1,2\% $(n=1)$ presentó deficiente $\mathrm{Cp}$ actividad en el grupo suplementado con $\mathrm{Fe}$ y $\mathrm{Fe}$ con $\mathrm{Zn}$, respectivamente. Al finalizar el estudio, la prevalencia de deficiencia de Cu no cambió con respecto a la del inicio.

Las participantes no mostraron diferencias en la ingesta de macro y micronutrientes al comparar por tipo de tratamiento. Sin embargo, presentaron una disminución significativa en la

\section{FIGURA 1}

Descripción de ensayo clínico, aleatorizado, controlado por placebo.

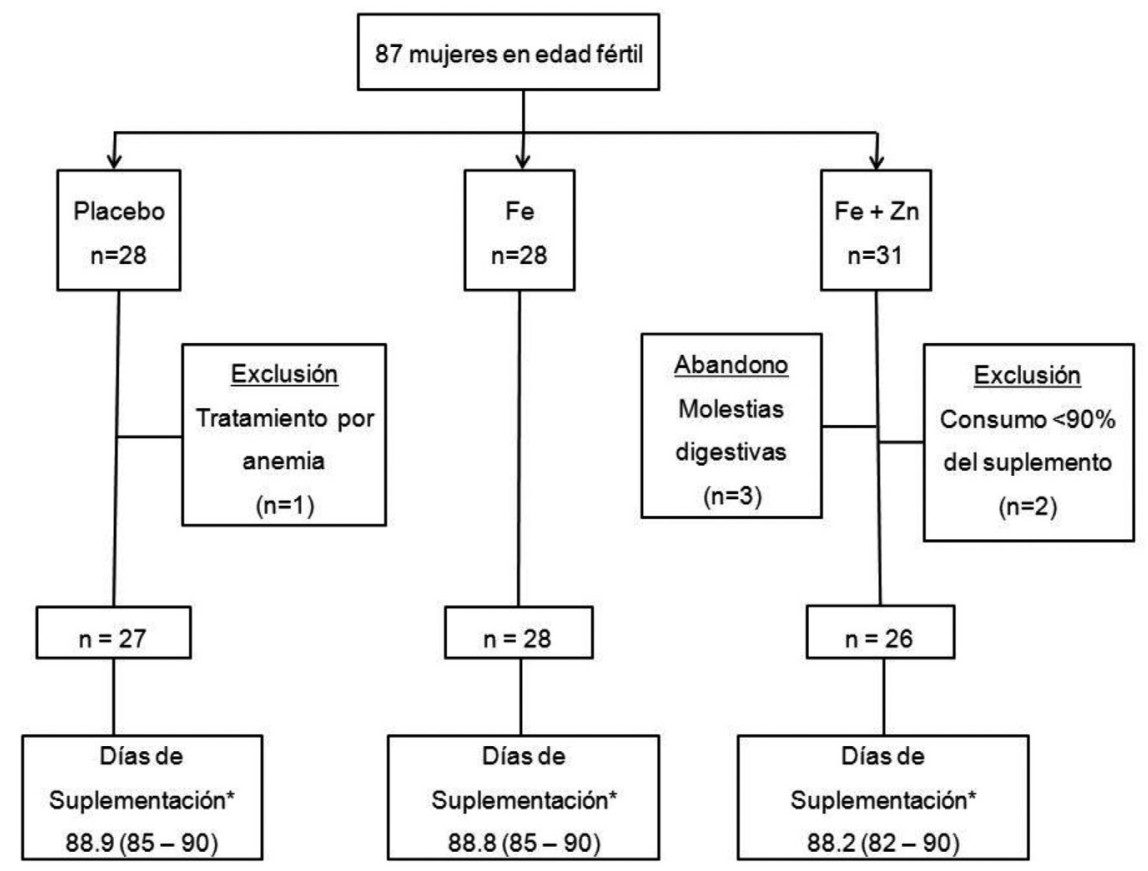

* Días promedio de ingesta del suplemento y rango (valor mínimo y máximo).

TABLA 1

Características de las participantes por grupo al inicio y después de la intervención.

\begin{tabular}{|c|c|c|c|c|c|c|c|c|c|}
\hline & \multicolumn{2}{|c|}{$\begin{array}{l}\text { Grupo Placebo } \\
\qquad(\mathrm{n}=27)\end{array}$} & \multicolumn{2}{|c|}{$\begin{array}{l}\text { Suplementación } \\
\text { con Hierro } \\
(n=28)\end{array}$} & \multicolumn{2}{|c|}{$\begin{array}{c}\text { Suplementación } \\
\text { Hierro-Zinc } \\
(n=26)\end{array}$} & \multirow{2}{*}{$\begin{array}{c}\text { Tiempo }{ }^{\mathrm{a}} \\
\mathrm{p}\end{array}$} & \multirow{2}{*}{$\begin{array}{c}\text { Tratamiento } \\
\text { p }\end{array}$} & \multirow{2}{*}{$\begin{array}{c}\text { Interacción } \\
\text { p }\end{array}$} \\
\hline & Inicio & Final & Inicio & Final & Inicio & Final & & & \\
\hline Edad (años) & $32,0 \pm 8,0$ & $32,2 \pm 8,0$ & $33,5 \pm 8,9$ & $33,9 \pm 9,0$ & $34,9 \pm 9,5$ & $35,0 \pm 9,5$ & 0,9 & 0,6 & 0,6 \\
\hline Talla (m) & $1,58 \pm 0,04$ & $1,57 \pm 0,05$ & $1,55 \pm 0,07$ & $1,56 \pm 0,05$ & $1,56 \pm 0,04$ & $1,57 \pm 0,05$ & 0,5 & 0,7 & 0,9 \\
\hline Peso (kg) & $67,3 \pm 11,1$ & $66.8 \pm 10,8$ & $68,5 \pm 9,7$ & $68,9 \pm 9,9$ & $66,6 \pm 8,8$ & $66,5 \pm 8,3$ & 0,8 & 0,7 & 0,5 \\
\hline $\mathrm{IMC}(\mathrm{kg} / \mathrm{m} 2)$ & $27,2 \pm 4,0$ & $27,0 \pm 4,1$ & $28,3 \pm 5,0$ & $28,3 \pm 4,5$ & $27,0 \pm 3,4$ & $26,9 \pm 3,2$ & 0,4 & 0,4 & 0,9 \\
\hline
\end{tabular}


ingesta de proteínas $(p<0,00)$ y $Z n(p<0,05)$ al comparar por tiempo (antes vs después) (tabla 3 )

En cuanto a la prevalencia de ingesta bajo el EAR de micronutrientes al inicio del estudio, el 1,2\% $(n=1)$ presentó ingesta insuficiente de Fe en los grupos suplementados con Fe y Fe con $\mathrm{Zn}$, respectivamente; el 1,2\% $(n=1)$ presentó deficiente ingesta de $\mathrm{Cu}$ en el grupo suplementado con $\mathrm{Fe}$; el
$1,2 \%(n=1), 2,5 \%(n=2)$ y $1,2 \%(n=1)$ una ingesta inadecuada de $\mathrm{Zn}$ en el grupo control, suplementado con Fe y Fe con $\mathrm{Zn}$. Al final, solo 1,2\% $(n=1)$ del grupo suplementado con Fe presentó inadecuación del consumo de éste, ninguna participante tuvo ingesta deficiente en $\mathrm{Cu}$ y el $2,5 \%(n=2)$ y $1,2 \%(n=1)$ pertenecientes al grupo control y suplementado con Fe, presentaron consumo deficiente de $\mathrm{Zn}$, respectivamente.

TABLA 2

Estado nutricional de cobre al inicio y después de la intervención.

\begin{tabular}{|c|c|c|c|c|c|c|c|c|c|}
\hline & \multicolumn{2}{|c|}{$\begin{array}{l}\text { Grupo Placebo } \\
\qquad(n=27)\end{array}$} & \multicolumn{2}{|c|}{$\begin{array}{l}\text { Suplementación } \\
\text { con Hierro } \\
(n=28)\end{array}$} & \multicolumn{2}{|c|}{$\begin{array}{c}\text { Suplementación } \\
\text { Hierro-Zinc } \\
(n=26)\end{array}$} & \multirow{2}{*}{$\begin{array}{c}\text { Tiempo }^{\mathrm{a}} \\
\mathrm{p}\end{array}$} & \multirow{2}{*}{$\begin{array}{c}\text { Tratamiento } \\
p\end{array}$} & \multirow{2}{*}{$\begin{array}{c}\text { Interacción } \\
\text { p }\end{array}$} \\
\hline & Inicio & Final & Inicio & Final & Inicio & Final & & & \\
\hline $\begin{array}{l}\text { Cp masa } \\
(\mathrm{mg} / \mathrm{L})^{*}\end{array}$ & $\begin{array}{c}533,5 \\
(419,7-678,4)\end{array}$ & $\begin{array}{c}539,3 \\
(410,9-708,8)\end{array}$ & $\begin{array}{c}516,0 \\
(405,8-656,9)\end{array}$ & $\begin{array}{c}566,0 \\
(442,2-673,4)\end{array}$ & $\begin{array}{c}521,5 \\
(418,2-649,8)\end{array}$ & $\begin{array}{c}566,0 \\
(429,1-747,4)\end{array}$ & 0,03 & 0,92 & 0,45 \\
\hline $\begin{array}{l}\text { Cp actividad } \\
(\mathrm{mg} / \mathrm{L})^{*}\end{array}$ & $\begin{array}{c}206,5 \\
(165,4-257,6)\end{array}$ & $\begin{array}{c}214,5 \\
(170,0-269,9)\end{array}$ & $\begin{array}{c}206,7 \\
(151,0-281,7)\end{array}$ & $\begin{array}{c}195,6 \\
(157,0-243,3)\end{array}$ & $\begin{array}{c}199,0 \\
(144,1-274,6)\end{array}$ & $\begin{array}{c}200,4 \\
(158,9-252,2)\end{array}$ & 0,92 & 0,62 & 0,47 \\
\hline $\begin{array}{l}\text { Índice Cp } \\
\text { actividad/ } \\
\text { Cp masa** }\end{array}$ & $0,402 \pm 0,12$ & $0,403 \pm 0,07$ & $0,41 \pm 0,11$ & $0,36 \pm 0,05$ & $0,39 \pm 0,08$ & $0,36 \pm 0,07$ & 0,02 & 0,38 & 0,18 \\
\hline $\begin{array}{l}\text { Cobre sérico } \\
(\mu \mathrm{g} / \mathrm{dL})^{* *}\end{array}$ & $135,8 \pm 45,2$ & $147,6 \pm 51,7$ & $125,7 \pm 33,3$ & $137,7 \pm 38,5$ & $143,5 \pm 52,0$ & $137,0 \pm 46,4$ & 0,00 & 0,87 & 0,01 \\
\hline $\begin{array}{l}\text { Abreviatura; } \\
\text { * Promedio } \\
\text { ** Media y d } \\
\text { a Antes vs de } \\
\text { ANOVA de }\end{array}$ & $\begin{array}{l}\text { eruloplasmina }(\mathrm{Cp} \\
\text { ométrico y, rango } \\
\text { viación estándar } \\
\text { ués } \\
\text { ías para medidas }\end{array}$ & $\begin{array}{l} \pm 1 \mathrm{DS} \\
\text { epetidas. }\end{array}$ & & & & & & & \\
\hline
\end{tabular}

\section{FIGURA 2}

Cambio (delta) de las concentraciones de cobre sérico post-suplementación.

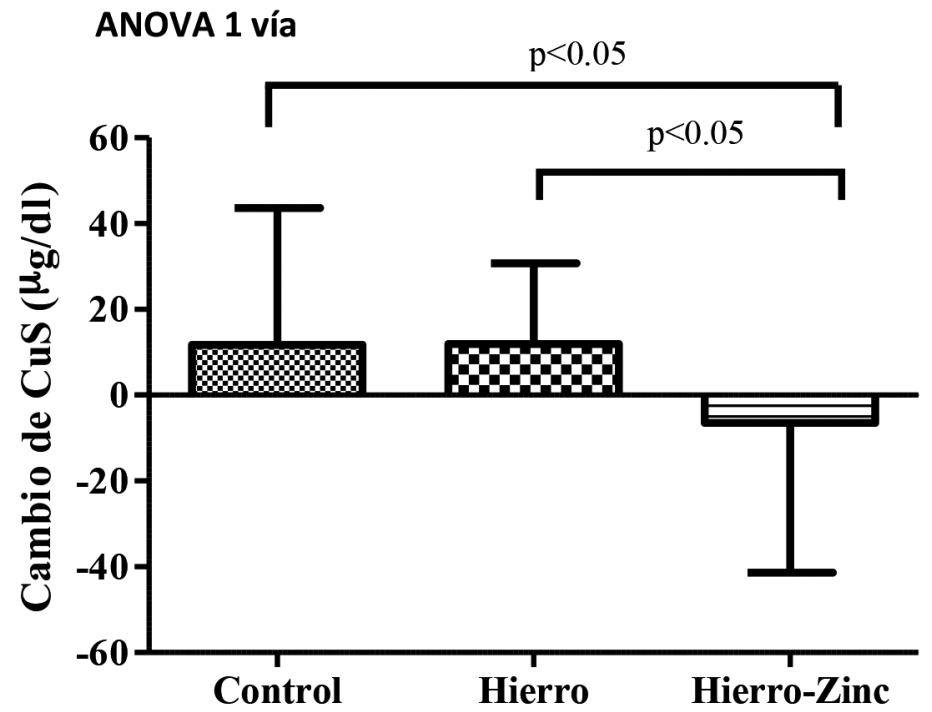




\section{DISCUSIÓN}

En este estudio observamos que la suplementación diaria con 30mg de Fe como sulfato ferroso por 3 meses no altera el estatus de $\mathrm{Cu}$ en mujeres de edad reproductiva, mientras que la suplementación diaria combinada con 30mg de Fe como sulfato ferroso y $30 \mathrm{mg}$ de $\mathrm{Zn}$ como sulfato de $\mathrm{Zn}$, disminuye significativamente la concentración de CuS.

Con respecto a la absorción y estatus de Cu, Domellöf et al. (9) evaluaron el efecto de la suplementación con $1 \mathrm{mg} / \mathrm{kg} /$ día de Fe elemental por 3 o 6 meses en lactantes menores de 1 año de ambos sexos, procedentes de Suiza y Honduras; solo aquellos procedentes de Honduras presentaron una disminución de la SOD1, pero ninguno mostró alteraciones en el CuS, sugiriendo que las reservas de $\mathrm{Cu}$ en los niños procedentes de Honduras pudieron haber estado depletadas al inicio del estudio y eso incidió en la alteración de SOD1 en dicho grupo. Cabe señalar que se ha descrito la disminución de esta enzima en otras condiciones, entre ellas en la deficiencia de Fe (22), por lo que se ha cuestionado su utilidad como un biomarcador exclusivo del estatus de $\mathrm{Cu}$ (23). A su vez, en una submuestra del mismo estudio, se observó que la suplementación con Fe a largo plazo (3 o 6 meses), no afecta la absorción de Cu en niños suizos (24). Este hallazgo fue similar al estudio realizado por Troost et al. en adultos con ileostomía, en el que se mostró que una dosis única de $20 \mathrm{mg}$ u $80 \mathrm{mg}$ de Fe elemental no altera la absorción de Cu.10 Dado que en nuestro estudio el Fe no alteró los biomarcadores de Cu, la disminución de CuS observada en el grupo con la suplementación combinada puede ser atribuida a la suplementación con $\mathrm{Zn}$.

Existe amplia controversia y limitada evidencia acerca del efecto de la suplementación con Zn sobre el estatus de Cu. Un estudio de Bonham (25) mostró que en hombres adultos sanos, la suplementación con $30 \mathrm{mg}$ de Zn, más una ingesta dietética promedio de 10mg, por 14 semanas, no alteró CuS, Cp masa, actividad y SOD1 de la misma forma que una suplementación con $20 \mathrm{mg}$ de $\mathrm{Zn}$ por 2 meses no alteró los indicadores de la nutrición de $\mathrm{Cu}$ en mujeres chilenas (26), mientras que en un estudio realizado en 387 adultos mayores, se observó que el CuS no se afectó con una suplementación con 15 o 30mg de Zn en hombres, sin embargo, sí disminuyó de forma significa- tiva en mujeres con una suplementación de 30mg.27 Por otra parte, Yadrick et al. (28), en un estudio realizado en 18 mujeres adultas sanas, mostró que la suplementación con 50mg de Zn o con éste mineral combinado con 50mg de Fe durante 10 semanas, disminuyeron las SOD1. En otro estudio de suplementación con 50mg de Zn en 26 hombres adultos sanos, se observó, igualmente, una reducción de SOD1 (29). A su vez se ha señalado que con dosis menores de $\mathrm{Zn}$ el estatus de $\mathrm{Cu}$ puede alterarse, según lo observado en púberes deportistas y adultos deportistas, brasileños, en los que disminuyeron las concentraciones de CuS después de una suplementación con $22 \mathrm{mg}$ de $\mathrm{Zn}$ por 12 y 4 semanas, respectivamente $(12,30)$. Estos estudios coincidieron con lo observado por Boukaïba et al. (13), en adultos mayores, principalmente de sexo femenino, en quienes la suplementación con $20 \mathrm{mg}$ de $\mathrm{Zn}$ por 8 semanas disminuyó las concentraciones de CuS independiente del estado nutricional que presentaran los sujetos, enflaquecidas o normales, estos estudios apoyan los resultados observados en nuestro estudio en los que en dosis menores o iguales al límite de ingesta segura (UL) de $40 \mathrm{mg}$ de $\mathrm{Zn}$ fijado por la IOM de EEUU (18), pueden afectar el estatus de Cu. Cabe señalar que la Autoridad Europea de Seguridad Alimentaria (AESA) definió como límite máximo de ingesta segura $25 \mathrm{mg}$ de $\mathrm{Zn}$ (31). En nuestro estudio se administró una dosis de 30mg de Zn más un promedio de ingesta de $11 \mathrm{mg}$, lo cual es equivalente al UL de la IOM de EEUU, pero superior al de AESA, con dicha dosis las concentraciones de CuS disminuyeron, lo que sugeriría que sería necesario revisar el UL de Zn establecido por la IOM.

Estudios en células intestinales Caco2 muestran el importante rol del transportador de metales divalentes (DMT1), en la absorción intestinal de Fe no hem (32) y de forma menos relevante en la absorción de Cu y Zn. (33). Por lo tanto, el Fe no hem es transportado principalmente por DMT1, el Cu por el transportador de Cu en humanos (Ctr1) y el Zn por ZIP4 (33). Dado que nosotros mostramos que la suplementación con Fe no afecta el estatus de Cu es posible que la interacción entre estos minerales por la vía de absorción intestinal, no sea biológicamente importante. Posibles explicaciones a la reducción de CuS observada en nuestro y otros estudios de suplementación con $\mathrm{Zn}$ puede atribuirse al hecho que el $\mathrm{Zn}$

TABLA 3

Ingesta de macro y micronutrientes por grupo al inicio y después de la intervención.

\begin{tabular}{|c|c|c|c|c|c|c|c|c|c|}
\hline & \multicolumn{2}{|c|}{$\begin{array}{l}\text { Grupo Placebo } \\
\qquad(n=27)\end{array}$} & \multicolumn{2}{|c|}{$\begin{array}{l}\text { Suplementación } \\
\text { con Hierro } \\
(n=28)\end{array}$} & \multicolumn{2}{|c|}{$\begin{array}{c}\text { Suplementación } \\
\text { Hierro-Zinc } \\
(n=26)\end{array}$} & \multirow{2}{*}{$\begin{array}{c}\text { Tiempo } \\
\text { p }\end{array}$} & \multirow{2}{*}{$\begin{array}{c}\text { Tratamiento } \\
\text { p }\end{array}$} & \multirow{2}{*}{$\begin{array}{c}\text { Interacción } \\
\text { p }\end{array}$} \\
\hline & Inicio & Final & Inicio & Final & Inicio & Final & & & \\
\hline Energía (kcal) & $2518,6 \pm 813,7$ & $2350,0 \pm 784,0$ & $2440,3 \pm 640,4$ & $2278,0 \pm 491,0$ & $2348,9 \pm 745,0$ & $2340,0 \pm 648,0$ & 0,07 & 0,8 & 0,7 \\
\hline Proteínas (g) & $102,6 \pm 33,6$ & $91,4 \pm 23,3$ & $101,5 \pm 33,5$ & $89,8 \pm 23,3$ & $100,8 \pm 34,1$ & $91,6 \pm 26,0$ & 0,002 & 0,9 & 0,9 \\
\hline Lípidos (g) & $50,8 \pm 25,1$ & $47,3 \pm 19,8$ & $45,0 \pm 17,0$ & $41,6 \pm 13,2$ & $44,0 \pm 19,5$ & $41,3 \pm 14,9$ & 0,1 & 0,2 & 0,9 \\
\hline $\mathrm{HC}(\mathrm{g})$ & $410,9 \pm 131,5$ & $387,0 \pm 129,0$ & $406,4 \pm 117,7$ & $388,3 \pm 88,8$ & $388,3 \pm 126,5$ & $403,0 \pm 121,9$ & 0,3 & 0,9 & 0,6 \\
\hline Cobre (mg) & $1,8 \pm 0,5$ & $1,9 \pm 0,7$ & $2,1 \pm 1,4$ & $1,6 \pm 0,5$ & $1,8 \pm 0,7$ & $1,8 \pm 0,8$ & 0,3 & 0,9 & 0,1 \\
\hline Hierro (mg) & $18,6 \pm 5,6$ & $17,8 \pm 6,4$ & $18,2 \pm 7,5$ & $16,7 \pm 4,8$ & $17,9 \pm 7,3$ & $18,3 \pm 7,9$ & 0,5 & 0,9 & 0,4 \\
\hline Zinc (mg) & $12,2 \pm 3,4$ & $11,6 \pm 3,8$ & $11,8 \pm 4,9$ & $10,2 \pm 3,0$ & $11,4 \pm 4,0$ & $10,7 \pm 3,1$ & 0,05 & 0,5 & 0,7 \\
\hline
\end{tabular}


induce la síntesis de metalotioneínas de novo, posiblemente de isoformas MT-1 y MT2, las cuales tienen mayor afinidad por Cu que por Zn (34), por lo tanto, una vez que Cu ingresa al enterocito las metalotioneínas lo secuestran impidiendo que se una a chaperonas, que son proteínas intracelulares que lo transportan hacia organelos, cuproenzimas y ATP7A, la cual se encarga de exportar el Cu a la circulación. El Cu unido a metalotioneína se elimina por las heces cuando el enterocito se descama (35). Por otra parte, se ha documentado que el incremento en la ingesta de Zn genera una sobreexpresión de DMT1 (36), lo que puede explicar que la absorción de Fe no se haya afectado y que probablemente la del cobre tampoco, sin embargo, dado a que pudiera haber un incremento en la síntesis de metaloproteínas inducido por Zn a nivel de enterocito, las pérdidas de cobre por las heces se incrementen y eso pueda explicar que el balance de $\mathrm{Cu}$ sea negativo en sujetos que ingieren más Zn (37). No podemos descartar que hubiera contribuido a ese efecto una competencia entre estos minerales por el transportador DMT1.

En este estudio llama la atención que no se alteraran las concentraciones de Cp masa, ya que está ampliamente descrita la correlación del CuS y la Cp masa $(38,39)$, no tenemos explicación para esta discrepancia. Las concentraciones de la $\mathrm{Cp}$ actividad disminuyeron, pero no de forma significativa, es posible que el tamaño muestral no haya sido suficiente para evidenciar un efecto significativo. Dado a la discrepancia en los efectos observados en CuS y Cp, aún no se puede concluir fehacientemente que la suplementación con Fe y $\mathrm{Zn}$ puede afectar la nutrición general del $\mathrm{Cu}$, ya que si así fuera, la concentración de Cp masa y actividad estarían alteradas como sucedió con CuS, son necesarios otros estudios que contribuyan a dilucidar esta interrogante.

Es importante destacar que la disminución de los niveles de CuS no cambió la prevalencia de deficiencia de $\mathrm{Cu}$, esto puede deberse a que el $\mathrm{Cu}$ tiene mecanismos altamente regulados que protegen contra deficiencia o exceso $(11,40)$, también es posible que la suplementación con Fe y $\mathrm{Zn}$ a las dosis estudiadas no afecta la absorción de $\mathrm{Cu}$, o bien, que afectando la absorción de este mineral, se necesitara una suplementación más prolongada para evidenciar un cambio significativo en la prevalencia debido a que las mujeres de nuestro estudio tenían una nutrición adecuada de este mineral.

En este estudio se observó una significancia estadística del tiempo sobre los niveles de CuS, Cp masa y la actividad específica de Cp, lo cual es consistente con otros estudios $(41,42)$. Varios factores pueden estar involucrados en esta variación, uno de ellos es la estacionalidad. Un estudio en Chile (43) mostró que la estacionalidad afecta los niveles séricos de micronutrientes, en nuestro estudio se observó que la ingesta de cobre y hierro no cambió entre invierno y primavera, por lo que la dieta no explicaría esta variación, es posible que otros cambios fisiológicos como los cambios hormonales dependientes de la exposición lumínica o variaciones del volumen plasmático debidos a la temperatura ambiental, puedan generar variaciones en los niveles de Cu (43).

Cabe destacar que la fortaleza de este estudio fue el ser un ensayo clínico, aleatorizado, doble ciego, controlado con placebo y con administración supervisada. Nosotros consideramos como una debilidad el no haber utilizado biomarcadores más sensibles de deficiencia leve y no haber tenido un grupo suplementado solo con Zn.

La importancia de la evaluación nutricional de Cu está dada por su rol en varios procesos metabólicos y las principales manifestaciones clínicas de su deficiencia son anemia, neutropenia y anormalidades óseas (42). La deficiencia de Cu tendría repercusiones en la homeostasis de $\mathrm{Fe}$, mediante la disminución de las concentraciones de ceruloplasmina, necesaria para oxidar al Fe y pasarlo a su estado férrico $(\mathrm{Fe}+3)$ para poder ser incorporado a la transferrina, proteína encargada de movilizarlo $(8,45)$.

Son necesarios más estudios que evalúen el efecto de la suplementación con minerales en dosis menores $y$, evaluar biomarcadores más sensibles (citocromo c oxidasa en plaquetas o leucocitos y/o en eritrocitos o mononucleares la CCS que es la chaperona de la SOD), para detectar deficiencia leve, y que contribuyan a dilucidar si la suplementación combinada con Fe y $\mathrm{Zn}$ a estas dosis constituye un riesgo para desarrollar deficiencia de Cu. Mientras se dilucida si la suplementación con Zn afecta el estatus de $\mathrm{Cu}$ se recomienda evaluar biomarcadores del estatus de cobre en sujetos que están siendo suplementados con éste mineral.

\section{CONCLUSIÓN}

La suplementación con 30mg de Fe combinada con 30mg de $\mathrm{Zn}$ disminuye de forma significativa la concentración de CuS en mujeres en edad reproductiva, mientras que la con $30 \mathrm{mg}$ de Fe solo no afecta el estatus de Cu en este grupo de la población.

\section{RESUMEN}

La deficiencia de hierro (Fe), cobre (Cu) y zinc ( $\mathrm{Zn}$ ) coexisten en países en vías de desarrollo. La implementación de programas de suplementación y fortificación con micronutrientes deben considerar posibles interacciones negativas en su absorción. El objetivo del estudio fue evaluar el efecto de la suplementación diaria de $30 \mathrm{mg}$ de Fe solo o combinado con $30 \mathrm{mg}$ de $\mathrm{Zn}$ sobre el estatus de $\mathrm{Cu}$ en mujeres chilenas en edad reproductiva. Este fue un ensayo clínico, doble ciego, aleatorizado y controlado por placebo, en el que 81 mujeres entre 18 y 45 años fueron asignadas al azar para recibir una diariamente, por tres meses, $30 \mathrm{mg}$ de Fe solo o combinado con $30 \mathrm{mg}$ de $\mathrm{Zn}$ o placebo. Al inicio y al final del estudio se evaluó la concentración de cobre sérico (CuS), ceruloplasmina (Cp masa), actividad de ceruloplasmina (Cp actividad) y actividad específica (Cp actividad/Cp masa). La suplementación diaria combinada con Fe y Zn disminuyó la concentración de CuS (ANOVA 2 vías para medidas repetidas $\mathrm{p}<0,01$ ), pero el Fe solo no afectó el estatus de Cu. La suplementación con Fe y Zn no modificó la Cp masa, Cp actividad y actividad específica. En conclusión, la suplementación combinada con 30mg de Fe y Zn disminuye significativamente las concentraciones de CuS.

Palabras clave: Cobre, hierro, zinc, suplementación, mujeres de edad reproductiva

Agradecimientos: Este estudio fue financiado por el proyecto Fondecyt número 1130075.

\section{BIBLIOGRAFÍA}

1. World Health Organization, Center for Disease Control and Prevention Atlanta. Worldwide prevalence of anaemia 1993-2005, WHO Global Database on Anaemia. Geneva, World Health Organization, 2008

2. Ramakrishan U. Prevalence of Micronutrient Malnutrition Worldwide. Nutr Rev. 2002; 60: S24 - S52.

3. International Zinc Nutrition Consultative Group (IZiNCG) Technical Document \#1. Assessment of Risk of Zinc Deficiency in Populations and Options for its Control. Hotz C, Brown KH, eds. Food Nutr Bull 2004; 25: S91 - S202 
4. Olivares M, Lera L, Albala C, Pizarro F, Araya M. Prevalencia de las deficiencias de zinc y cobre en adultos mayores de la Región Metropolitana de Santiago. Rev Med Chile 2011; 139: $283-289$.

5. Olivares M, Walter T, Hertrampf E, Pizarro F. Anaemia and iron deficiency disease in children. Brit Med Bull. 1999; 55: $534-543$

6. Solomons $N$, Ruz M. Zinc and iron interaction: concepts and perspectives in the developing world. Nutr Res. 1997; 17: $177-185$

7. Olivares M, Pizarro F, Hertramp E, Walter T, Arredondo M, Letelier A. Fortificación de alimentos con hierro en Chile. Rev Chil Nutr. 2000; 27: 340 - 344

8. Lönerdal B. Iron-zinc-copper Interactions. In: Agency for international Development Food and Agriculture Organization of the United Nation editor. Micronutrient interactions Impact on child health and nutrition. Washington: International Life Science Institute Press; 1998: 3 - 10.

9. Domellöf M, Dewey $K$, Cohen R, Lönnerdal B, Hernell O. Iron supplements reduce erythrocyte copper - zinc superoxide dismutase activity in term, breast infants. Acta Paediatr 2005; 94: 1578 - 1582.

10. Troost F, Brummer RJ, Dainty J, Hoogewerff J, Bull V, Saris $W$. Iron supplements inhibit zinc but not copper absorption in vivo in ileostomy subjects. Am J Clin Nutr 2003; 78: 1018 - 1023 .

11. López de Romaña D, Olivares M, Uauy R, Araya M. Risks and benefits of copper in light of new insights of copper homeostasis. J Trace Elem Med Biol 2011; 25: 3 - 13.

12. Fernandes $K$, Marino C, Vianna A, Porto C, Correa J. Effect of zinc supplementation on the antioxidant, copper, and iron status of physically active adolescents. Cell Biochem Funct 2009; 27: 162 - 6.

13. Boukaïba N, Flament C, Acher S, Chappuis P, Piau A, Fusselier M, Dardenne M, Lemonnier D. A physiological amount of zinc supplementation: effects on nutritional, lipid, and thymic status in an elderly population. Am J Clin Nutr. 1993; 57: $566-72$.

14. Norton K, Whittingham N, Carter L, Kerr D, Gore C, Marfell-Jones M. Técnicas de medición en antropometría. In: Norton K, Olds T editors. Antropometrica (Edición en español: Mazza JC) Argentina: Biosystem Servicio Educativo Press; 2000: 23 - 60.

15. Schmidt-Hebbel H, Pennacchiotti I, Masson L, Mella MT. Tabla de composición química de los alimentos chilenos. Santiago, Chile: Facultad de Ciencias Químicas y Farmacéuticas, Departamento de Ciencia de los Alimentos y Tecnología Química, Universidad de Chile, 1992.

16. Olivares M, Pizarro F, De Pablo S, Araya M, Uauy R. Iron, zinc and copper: contents in common Chilean foods and daily intakes in Santiago City, Chile. Nutrition 2004; 20: $205-12$

17. United States Department of Agriculture. USDA national nutrient database for standard reference, release 24; 2012. Accesada online el 21 Enero 2014 en http://www.ars.usda. gov/Services/docs.htm?docid $=22808$

18. Institute of Medicine (IOM), Food and Nutrition Board. Dietary Reference Intakes for Vitamin A, Vitamin K, arsenic, boron, chromium, copper, iodine, iron, manganese, molybdenum, nickel, silicon, vanadium, and zinc. Washington, DC: The National Academies Press; 2001.

19. Ravin A. An improved colorimetric enzymatic assay for ceruloplasmin. J Lab Clin Med 1961; 58: 161 - 168.

20. Elin RJ. Reference intervals and laboratory values. In: Cecil
Textbook of Medicine. 22th ed. Goldman L, Ansiello DA, editors. WB Saunders, Philadelphia, PA; 2004: 2496-2505.

21. Cediel G, Olivares M, Araya M, Letelier A, López de Romaña $D$, Pizarro F. Efecto de la inflamación subclínica sobre el estado nutricional de hierro, cobre y zinc en adultos. Rev Chil Nutr. 2009; 36: 8 - 14.

22. Olivares M, Araya M, Pizarro F, Letelier A. Erythrocyte CuZn superoxide dismutase activity is decreased in iron-deficiency anemia. Biol Trace Elem Res. 2006; 112: 213 - 220

23. Milne D, Davis C, Nielsen H. Low dietary zinc alters indices of copper function and status in postmenopausal women. Nutrition 2001; 17: $701-708$

24. Domellöf M, Hernell O, Abrams SA, Chen Z, Lönnerdal $B$. Iron supplementation does not affect copper and zinc absorption in breastfed infants. Am J Clin Nutr 2009; 89: $185-90$

25. Bonham M, O'Connor J, Mcanena, Walsh P, Downes SP, Hannigan B, Stran JJ. Zinc Supplementation has no effect on lipoprotein metabolism, hemostasis, and putative indices of copper status in healthy men. Biol Trace Elem Res 2003; 93: $75-86$

26. Lopez de Romaña D, Ruz M, Pizarro F, Landaeta L, Olivares $M$. Supplementation with zinc between meals has no effect on subsequent iron absorption or iron status of Chilean women. Nutrition 2008; 24: 957 - 63.

27. Hininger - Favier I, Andriollo - Sanchez M, Arnaud J, Meunier N, Bord S, Graham C, Polito A, Maiani G, O'Connor J, Coudray C, Roussel AM. Age- and sex-dependent effects of long-term zinc supplementation on essential trace element status and lipid metabolism in European subjects: the Zenith Study. Brit J Nutr 2007; 97: 569 - 78.

28. Yadrick $K$, Kenny $A$, Winterfeldt $A$. Iron, copper, and zinc status: response to supplementation with zinc or zinc and iron in adult females. Am J Clin Nutr 1989; 49: 145 - 150.

29. Fischer F, Giroux A, L'Abbé L. Effect of zinc supplementation on copper status in adult man. Am J Clin Nutr 1984; 40: $743-46$.

30. Fernandes L, Marino C, Gastao J, Pires L, Severino A, Casimiro Lopes G, Lisboa P, Correa J. Plasma Zinc, copper and serum thyroid hormones and insulin levels after zinc supplementation followed by placebo in competitive athletes. Biol Trace Elem Res 2011; 142: 415 - 23.

31. European Food Safety Authority: Scientific Committee on Food, Scientific Panel on Dietetic Products, Nutrition and Allergies. Opinion of the Scientific Committe on Food on the Tolerable upper intake level of zinc. In: Tolerable upper intake levels for vitamins and minerals; 2006: $p 191-201$.

32. Arredondo M, Muñoz P, Mura CV, Núñez MT. DMT1, a physiologically relevant apical Cu1 transporter of intestinal cells. Am J Physiol Cell Physiol 2003; 284: C1525 - C30.

33. Espinoza A, Le Blanc S, Olivares M, Pizarro F, Ruz M, Arredondo $M$. Iron, copper, and zinc transport: inhibition of Divalent Metal Transporter 1 (DMT1) and Human Copper Transporter 1 (hCTR1) by shRNA. Biol Trace Elem Res 2012; 146: $281-6$

34. Waalkes $M$, Harvey $M$, Klaassen $C$. Relative in vitro affinity of hepatic metallothionein for metals. Toxicol Lett 1984; 20: 33 - 9.

35. Olivares $M$, Pizarro F, López de Romaña D, Arredondo $M$. Absorción y transporte de microminerales. In: Brunser $O$, Cruchet S, Gotteland M, eds. Fisiología gastrointestinal y nutrición. Chile: Nestlé Chile Press; 2013: 133 - 49.

36. lyengar V, Pullakhandam R, Madhavan K. Coordinate expression and localization of iron and zinc transporters 
explain iron-zinc interactions during uptake in Caco-2 cells: implications for iron uptake at the enterocyte. J Nutr Biochem 2012; 23: 1146 - 54.

37. Festa M, Anderson H, Dowdy R, Ellersieck M. Effect of zinc intake on copper excretion and retention in men. Am J Clin Nutr 1985; 41: 285 - 92.

38. Olivares M, Méndez M, Astudillo P, Pizarro F. Present situation of biomarkers for copper status. Am J Clin Nutr 2008; 88: 859S - 62S.

39. Arredondo M, Gonzalez M, Olivares M, Pizarro F, Araya M. Ceruloplasmin, an indicator of copper status. Biol Trace Elem Res 2008; 123:261-9

40. Linder C, Hazegh-Azam M. Copper biochemestry and molecular biology. Am J Clin Nutr 1996; 63: 797S-811S.
41. Araya M, Pizarro F, Olivares M, Arredondo M, González M, Méndez M. Understanding copper homeostasis in humans and copper effects on health. Biol Res 2006; 39: $183-87$.

42. Wiedeman A, Olivares M, Pizarro F, Araya M. Suplementación con cobre entre comidas no tiene efecto sobre la nutrición de hierro en hombres. Rev Chil Nutr 2009; 36: 1114 - 9.

43. Miranda M, Olivares $M$, Araya M, Pizarro F. Efecto de la estacionalidad sobre el estado nutricional de hierro, zinc $y$ cobre. Rev Chil Nutr 2013; 40: 107 - 11.

44. Olivares M, Uauy R. Copper as an essential nutrient. Am J Clin Nutr 1996; 63: 791S - 796S.

45. Collins F, Prohaska R, Knutson D. Metabolic crossroads of iron and copper. Nutr Rev 2010; 68:133 - 47. 